\title{
Corrigendum: Identification and Analysis of the Blood IncRNA Signature for Liver Cirrhosis and Hepatocellular Carcinoma
}

Qi Xia ${ }^{1,2,3 * t}$, Zheyue Shu ${ }^{3,4,5 t}$, Ting Ye ${ }^{3}$ and Min Zhang ${ }^{3,4,5 *}$

${ }^{1}$ State Key Laboratory for Diagnosis and Treatment of Infectious Diseases, National Clinical Research Center for Infectious Diseases, Collaborative Innovation Center for Diagnosis and Treatment of Infectious Diseases, The First Affiliated Hospital, College of Medicine, Zhejiang University, Hangzhou, China, ${ }^{2}$ Key Laboratory for Biomedical Engineering of Ministry of Education, Zhejiang University, Hangzhou, China, ${ }^{3}$ Zhejiang University, Hangzhou, China, ${ }^{4}$ Division of Hepatobiliary and Pancreatic Surgery, Department of Surgery, The First Affiliated Hospital, College of Medicine, Zhejiang University, Hangzhou, China, ${ }^{5}$ Key Laboratory of Combined Multi-Organ Transplantation, Ministry of Public Health, Hangzhou, China

Keywords: hepatocellular carcinoma, hepatitis B virus, IncRNA, liver cirrhosis, support vector machine

\section{OPEN ACCESS}

Approved by: Frontiers in Editorial Office, Frontiers Media SA, Switzerland

${ }^{*}$ Correspondence:

QiXia

xiaqi@zju.edu.cn

Min Zhang

zhangminnn296@163.com

tThese authors have contributed equally to this work

Specialty section: This article was submitted to

RNA

a section of the journal

Frontiers in Genetics

Received: 08 April 2021 Accepted: 12 May 2021 Published: 01 July 2021

Citation:

Xia Q, Shu Z, Ye T and Zhang M (2021) Corrigendum: Identification and

Analysis of the Blood IncRNA

Signature for Liver Cirrhosis and Hepatocellular Carcinoma.

Front. Genet. 12:692400.

doi: 10.3389/fgene.2021.692400

\section{A Corrigendum on}

Identification and Analysis of the Blood lncRNA Signature for Liver Cirrhosis and Hepatocellular Carcinoma

by Xia, Q., Shu, Z., Ye, T., and Zhang, M. (2021). Front. Genet. 11:595699. doi: $10.3389 /$ fgene.2020.595699

In the published article, there was an error in affiliation ${ }^{* *} 1^{* *}$. Instead of "*** Department of Infectious Disease, State Key Laboratory for Diagnosis and Treatment of Infectious Diseases, National Clinical Research Center for Infectious Diseases, Collaborative Innovation Center for Diagnosis and Treatment of Infectious Diseases, The First Affiliated Hospital, Zhejiang University School of Medicine, Hangzhou, China, ${ }^{* * "}$ it should be "***State Key Laboratory for Diagnosis and Treatment of Infectious Diseases, National Clinical Research Center for Infectious Diseases, Collaborative Innovation Center for Diagnosis and Treatment of Infectious Diseases, The First Affiliated Hospital, College of Medicine, Zhejiang University, Hangzhou, China. ${ }^{* *}$ "

Also, authors "Zheyue Shu" and "Min Zhang" were missing an affiliation: "*** Division of Hepatobiliary and Pancreatic Surgery, Department of Surgery, The First Affiliated Hospital, College of Medicine, Zhejiang University, Hangzhou, China. ${ }^{* *}$ Aforementioned corrections have been made and consequently, all affiliation numbers and order have been revised.

The authors apologize for this error and state that this does not change the scientific conclusions of the article in any way. The original article has been updated.

Copyright (c) $2021 \mathrm{Xia}$, Shu, Ye and Zhang. This is an open-access article distributed under the terms of the Creative Commons Attribution License (CC BY). The use, distribution or reproduction in other forums is permitted, provided the original author(s) and the copyright owner(s) are credited and that the original publication in this journal is cited, in accordance with accepted academic practice. No use, distribution or reproduction is permitted which does not comply with these terms. 